\title{
Résumé des recommandations sur le diagnostic et le traitement du paludisme du Comité consultatif de la médecine tropicale et de la médecine des voyages (CCMTMV)
}

\author{
Boggild A. ${ }^{1}$, Brophy J. ${ }^{2}$, Charlebois P. ${ }^{3}$, Crockett M. ${ }^{4}$, Geduld J. ${ }^{5}$, Ghesquiere W. ${ }^{6}$, McDonald P. ${ }^{7}$, \\ Plourde $P{ }^{8}$, Teitelbaum $P .{ }^{9}$, Tepper $M .^{10}$, Schofield $S^{11}$ et McCarthy A. (présidente) $)^{12^{\star}}$
}

1 University Health Network, Toronto General Hospital (Toronto, Ont.)

2 Division des maladies infectieuses, Centre hospitalier pour enfants de l'est de l'Ontario (Ottawa, Ont.)

3 Médecine interne, Centre des services de santé des Forces canadiennes (Atlantique) (Halifax, N.-É.)

4 Pédiatrie et santé des enfants, Université du Manitoba (Winnipeg, Man.)

5 Direction générale de la prévention et du contrôle des maladies infectieuses, Agence de la santé publique du Canada (Ottawa, Ont.)

6 Infectious Diseases and Internal Medicine, Université de la Colombie-Britannique (Victoria, C.-B.)

7 Direction des produits thérapeutiques, Santé Canada (Ottawa, Ont.)

8 Faculté de médecine, Université du Manitoba (Winnipeg, Man.)

${ }^{9}$ Clinique santé-voyage Riverside (Ottawa, Ont.)

${ }^{10}$ Programme de lutte contre les maladies transmissibles, Direction de la protection de la santé de la Force (Ottawa, Ont.)

${ }^{11}$ Entomologie de la lutte antiparasitaire, Direction de la protection de la santé de la Force (Ottawa, Ont.)

${ }^{12}$ Clinique de médecine tropicale et de santé internationale, Division des maladies infectieuses, Campus général de l'Hôpital d'Ottawa (Ottawa, Ont.)

* Auteure-ressource : AMcCARTHY@Ottawahospital.on.ca

\section{Résumé}

Contexte : Au nom de l'Agence de la santé publique du Canada, le CCMTMV a formulé les Recommandations canadiennes pour la prévention et le traitement du paludisme (malaria) chez les voyageurs internationaux à l'intention des fournisseurs de soins de santé qui préparent les patients qui voyageront dans des régions impaludées et qui traitent les voyageurs qui sont revenus malades au pays. L'objectif de ces recommandations consiste à effectuer un diagnostic et un traitement appropriés du paludisme, une maladie qui demeure peu connue au Canada.

Objectif : Fournir des recommandations sur le diagnostic et le traitement appropriés du paludisme.

Méthodologie : Le CCMTMV a examiné toutes les sources principales de renseignements sur le diagnostic et le traitement du paludisme, de même que les recherches récentes et les données épidémiologiques nationales et internationales afin de proposer des lignes directrices adaptées au contexte canadien. II a formulé ses recommandations dans le cadre d'une approche de la médecine fondée sur les preuves à l'aide d'échelles d'évaluation afin de déterminer le poids et la qualité des preuves.

Recommandations : La prise en charge du paludisme repose sur l'identification rapide de la maladie ainsi que sur l'identification de l'espèce et le dosage de la parasitémie. L'identification microscopique des échantillons sanguins est rapide et précise, mais ne peut être effectuée que par des techniciens de laboratoire qualifiés. Les tests diagnostiques rapides sont très répandus, simples à utiliser et ne nécessitent aucun équipement de laboratoire spécialisé ni aucune formation. Cependant, ils ne permettent pas de déterminer l'ampleur de la parasitémie et doivent être vérifiés. La réaction en chaîne de la polymérase $(R C P)$, bien que son accessibilité soit encore limitée, est de plus en plus reconnue comme l'étalon de référence à cause de sa grande sensibilité et de sa grande spécificité pour identifier l'espèce. 
Toute personne atteinte de paludisme grave ou compliqué doit être hospitalisée pour que sa fréquence et son profil respiratoires, son score de coma, sa glycémie et son débit urinaire soient régulièrement surveillés, en particulier si le patient est inconscient. En cas de forte parasitémie, la transfusion d'échange (exsanguinotransfusion) peut se révéler bénéfique pour éliminer les érythrocytes infectés ou les médiateurs toxiques de la circulation et pour réduire la charge parasitaire. En raison du risque élevé de paludisme grave ou compliqué, les personnes souffrant de Plasmodium falciparum doivent aussi être admises à l'hôpital ou recevoir un traitement initial dans une unité d'observation.

Le traitement du paludisme non compliqué vise à guérir l'infection et prévenir la progression vers la maladie grave. Lors du choix des régimes thérapeutiques, il convient de tenir compte de la tolérabilité au médicament, du profil d'effets indésirables et de la rapidité de la réponse thérapeutique.

\section{Introduction}

Le paludisme est une maladie infectieuse grave causée par cinq différentes espèces de Plasmodium :

$P$. falciparum, $P$. vivax, $P$. ovale, $P$. malariæ et $P$. knowlesi. Le paludisme est transmis par la morsure d'un moustique anophèle femelle infecté. Les infections à $P$. falciparum sont associées aux taux de mortalité les plus élevés. Le taux de mortalité global du paludisme à $P$. falciparum varie d'environ $1 \%$ à $5 \%$ et grimpe pour atteindre $20 \%$ dans les cas graves (1-2).

D'après l'Organisation mondiale de la Santé (OMS), environ 3,3 milliards de personnes dans le monde couraient un risque de paludisme en 2010; on a ainsi enregistré environ 219 millions de cas, dont $80 \%$ dans 17 pays et approximativement $40 \%$ en Inde, au Nigeria et en République démocratique du Congo (3). Le paludisme est encore diagnostiqué au Canada au retour de voyages dans les pays où la maladie est endémique. Le Système canadien de surveillance des maladies à déclaration obligatoire, qui surveille les maladies infectieuses à déclaration obligatoire à l'échelle du pays, a été informé de 4254 cas de paludisme entre 2001 et 2011 (D. Taylor, Agence de la santé publique du Canada, données inédites, 2013).

D'août 2001 à août 2012, le Réseau canadien sur le paludisme, qui permet d'accéder rapidement au traitement parentéral du paludisme grave, a été averti de l'existence de 195 cas de paludisme grave ou compliqué (comm. pers., A. McCarthy et J. Geduld, CCMTMV, 2012).

Les facteurs déterminants pour la survie des patients sont un diagnostic précoce et un traitement approprié. Les décès causés par le paludisme peuvent pour la plupart être évités et sont souvent attribuables aux retards dans le diagnostic et le traitement. Parmi les cas rapportés au Réseau canadien sur le paludisme, seulement $20 \%$ des personnes atteintes se sont présentées à un établissement de soins de santé dans les 24 heures à compter de l'apparition des symptômes et $44 \%$ ont attendu plus de trois jours (communication personnelle, A. McCarthy et J. Geduld, ainsi que K. Cullen et P. Arguin des Centers for Disease Control and Prevention [États-Unis], 2012). Le diagnostic par le fournisseur de soins de santé a été retardé de plus de 24 heures dans $34 \%$ des cas (A. McCarthy et J. Geduld, comm. pers., 2012).

II s'agit ici d'un résumé des Recommandations canadiennes pour la prévention et le traitement du paludisme (malaria) chez les voyageurs internationaux, du CCMTMV, formulées à l'intention des fournisseurs de soins de santé qui préparent les patients qui voyageront dans des régions impaludées et qui traitent les voyageurs qui sont revenus malades au pays (4). Ces lignes directrices comprennent une description complète des recommandations sur le diagnostic et le traitement du paludisme. 


\section{Méthodologie}

Le sous-comité sur le paludisme, un groupe de travail du CCMTMV, a élaboré les lignes directrices. Le processus mis en place pour les concevoir a été décrit précédemment (5). II comprenait un examen des recherches récentes et des données épidémiologiques nationales et internationales ainsi que la prise en compte d'autres facteurs, comme l'épidémiologie du paludisme, ainsi que les valeurs et les préférences des voyageurs et des fournisseurs de soins de santé. Les recommandations dans le cadre d'une approche de la médecine fondée sur les preuves pour le diagnostic et le traitement du paludisme ont été formulées à l'aide d'échelles d'évaluation afin de déterminer le poids et la qualité des preuves.

\section{Recommandations}

Les recommandations du CCMTMV dans le cadre d'une approche de la médecine fondée sur les preuves en ce qui concerne le diagnostic et le traitement du paludisme sont résumées dans le Tableau 1. Une analyse de certaines recommandations principales suivra.

Tableau 1: Recommandations dans le cadre d'une approche de la médecine fondée sur les preuves en ce qui concerne le diagnostic et le traitement du paludisme

\section{Recommandation}

1. L'artésunate parentéral est recommandé comme traitement de première intention du paludisme à $P$. falciparum grave, tandis que la quinine parentérale est recommandée comme traitement de remplacement (3).

2. Du phosphate de primaquine (30 mg [base] par jour pendant 2 semaines) devrait être administré après un traitement par la chloroquine pour prévenir les rechutes du paludisme à $P$. vivax ou à $P$. ovale (6).

3. Les traitements de choix du paludisme à $P$. falciparum non compliqué consistent en l'administration de :

- chloroquine par voie orale (SEULEMENT chez les sujets ayant voyagé dans une région où les souches sont sensibles uniquement à la chloroquine);

- atovaquone/proguanil par voie orale (7);

- quinine orale combinée à de la doxycycline ou de la clindamycine par voie orale;

- $\quad$ polythérapie par un dérivé de l'artémisinine (non encore accessible au Canada) (7).

4. La transfusion d'échange peut être utile pour traiter les cas de paludisme à $P$. falciparum hyperparasitémique (8).

5. Les personnes se rendant dans une région où le paludisme est sensible à la chloroquine devraient amorcer l'auto-traitement à la chloroquine, puis continuer ou amorcer une prophylaxie à la chloroquine (9-11).

6. Dans les régions où $P$. falciparum est résistante à la chloroquine ou à la chloroquine et à la méfloquine, l'auto-traitement devrait être une solution de rechange aux médicaments utilisés à titre prophylactique; on choisira l'une des options suivantes :

a. atovaquone/proguanil (Malarone ${ }^{\mathrm{MD}}$ );

b. quinine et doxycycline orales;

c. artéméther/luméfantrine $\left(\right.$ Coartem $\left.{ }^{\mathrm{MD}}\right)$, idéalement acheté dans un pays où les normes pharmaceutiques sont rigoureuses (p. ex. en Europe ou aux États-Unis) afin de réduire au minimum le risque d'utiliser un produit contrefait (10-13). 

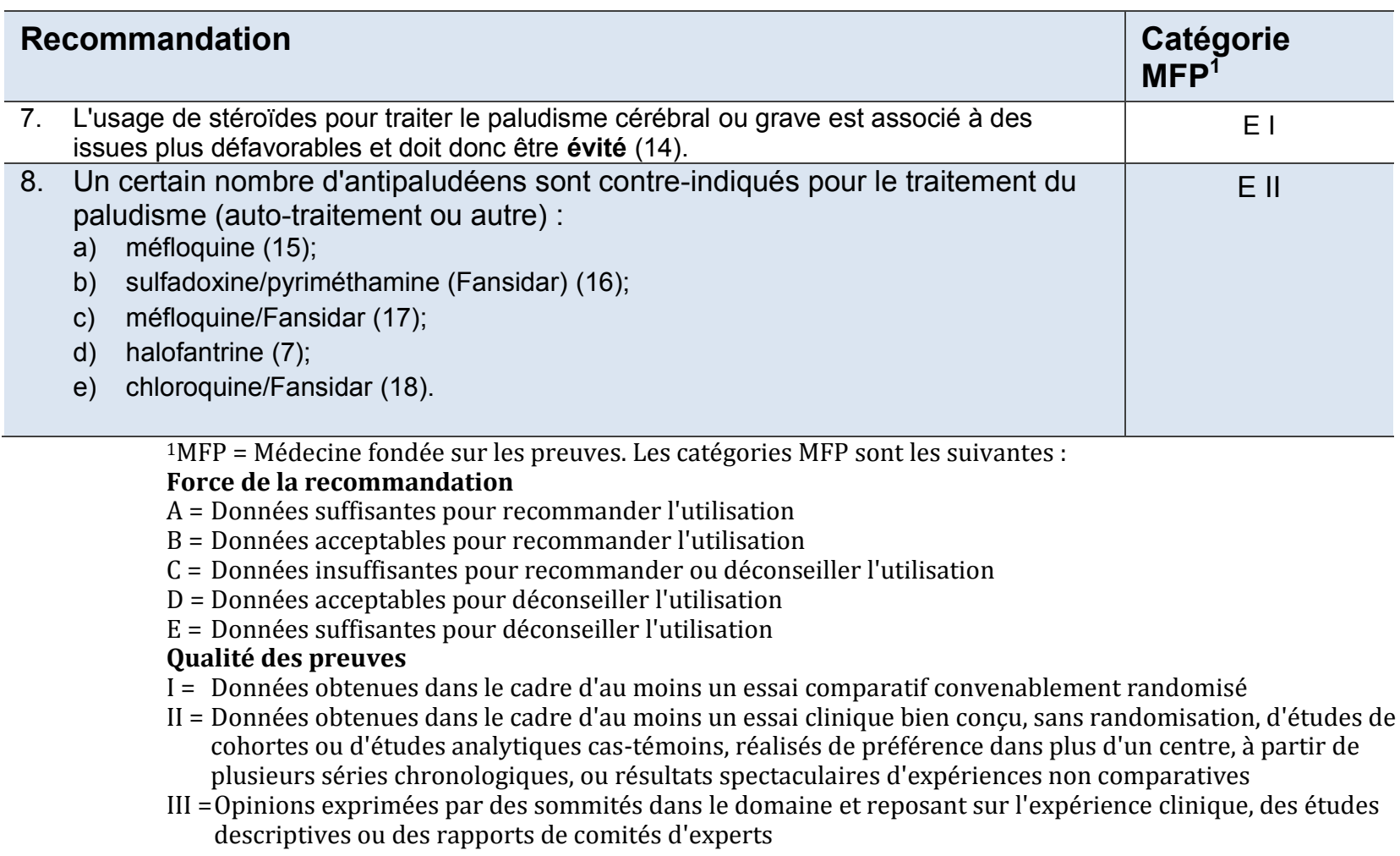

\section{Diagnostic du paludisme}

Le paludisme pourrait expliquer l'apparition d'une fièvre dont la cause est inconnue qui se manifeste chez un voyageur en visite dans une région impaludée ou jusqu'à un an après son départ d'une telle région, indépendamment de l'utilisation d'une chimioprophylaxie (19). Si le voyageur présente de la fièvre durant cette période, il doit se faire examiner immédiatement par un médecin et l'informer de ses antécédents de voyage. II faut être particulièrement attentif aux fièvres qui se déclarent dans les trois mois suivant le retour d'un voyage, car plus de $90 \%$ des cas de paludisme à $P$. falciparum surviennent au cours de cette période.

Puisque la maladie peut évoluer de l'infection asymptomatique à la maladie grave, avec complications, et même entraîner la mort en l'espace de 36 à 48 heures, la survie des patients ayant contracté le paludisme, particulièrement le paludisme à $P$. falciparum (20), repose sur l'établissement d'un diagnostic précoce et la nécessité de bien identifier l'espèce afin de choisir le traitement adéquat qui pourra sauver leur vie. Le dosage de la parasitémie est également important pour déterminer si un traitement parentéral, une transfusion d'échange ou une admission à l'unité de soins intensifs sont nécessaires. De plus, il est important de surveiller la réponse au traitement.

L'examen microscopique de frottis sanguins (étalement mince et goutte épaisse) est à la fois rapide et précis. Un laboratoire canadien devrait être en mesure de confirmer la présence du parasite et, dans la plupart des cas, de déterminer l'espèce en cause, moins de 1 à 2 heures après la réception d'un échantillon sanguin (21-24). II faut toutefois beaucoup de formation et d'expérience pour bien analyser les frottis sanguins $(23,25)$.

Les tests diagnostiques rapides sont simples à utiliser et n'exigent pas de matériel de laboratoire spécialisé ni de compétences particulières. Ce sont des outils diagnostiques essentiels si l'on ne peut obtenir les résultats de la microscopie pour le paludisme en moins de 2 heures (26). Cependant, les résultats positifs ou négatifs doivent être vérifiés par microscopie spécialisée ou réaction de polymérisation en chaîne (RCP) pour déterminer 
l'ampleur de la parasitémie et identifier l'espèce. Veuillez noter que les tests diagnostiques rapides utilisés par les voyageurs pour établir eux-mêmes un diagnostic ne sont pas fiables.

Bien que son accessibilité soit encore limitée, la RCP est de plus en plus reconnue comme l'étalon de référence à cause de sa grande sensibilité et de sa grande spécificité pour identifier l'espèce. Son utilisation ne cesse de croître pour le contrôle de la qualité (27-29).

\section{Principes généraux de la prise en charge du paludisme}

Lors de la prise en charge du paludisme, il convient de répondre à trois questions :

\section{1) Cette infection est-elle causée par $P$. falciparum?}

Le traitement varie selon l'espèce de plasmodies en cause. $P$. falciparum peut menacer le pronostic vital d'un hôte non immun et est une urgence médicale. Envisager d'hospitaliser tous les sujets non immuns et tous les enfants pour s'assurer qu'ils tolèrent les antipaludéens et pour détecter les complications ou un échec thérapeutique précoce.

\section{2) S'agit-il de paludisme grave ou compliqué?}

En présence de $P$. falciparum et en l'absence de toute autre cause évidente expliquant les symptômes, l'observation d'une ou plusieurs des caractéristiques cliniques ou biologiques suivantes indique qu'il s'agit d'un cas de paludisme grave ou compliqué :

\begin{tabular}{|l|l|}
\hline Manifestation clinique & Analyse de laboratoire \\
\hline Prostration/troubles de la conscience & Anémie grave $(\mathrm{Hct}<15 \% ; \mathrm{Hb} \leq 50 \mathrm{~g} / \mathrm{L})$ \\
\hline Détresse respiratoire & Hypoglycémie $($ glycémie $<2,2 \mathrm{mmol} / \mathrm{L})$ \\
\hline Convulsions multiples & Acidose $(\mathrm{pH}$ artériel $<7,25$ ou bicarbonate $<15 \mathrm{mmol} / \mathrm{L})$ \\
\hline Collapsus cardiovasculaire & Insuffisance rénale $($ créatinine $>265 \mu \mathrm{mol} / \mathrm{L})(1)$ \\
\hline OEdème pulmonaire (examen radiologique) & Hyperlactatémie \\
\hline Saignement anormal & Hyperparasitémie $(\geq 2 \%)$ \\
\hline Ictère & - \\
\hline Hémoglobinurie & - \\
\hline Adapté à partir des Directives pour le traitement du paludisme, Organisation mondiale de la Santé, 2010 (7). \\
\hline
\end{tabular}

Le paludisme grave est habituellement attribuable à une infection à $P$. falciparum, bien qu'il puisse être aussi causé par $P$. knowlesi. De plus, $P$. vivax peut parfois être à l'origine du paludisme grave.

\section{3) Où l'infection a-t-elle été contractée?}

L'annexe I des Recommandations canadiennes pour la prévention et le traitement du paludisme (malaria) chez les voyageurs internationaux montre une caractérisation régionale et pays par pays des zones de transmission du paludisme (4). Les parasites du paludisme de presque partout dans le monde sont résistants aux médicaments. En cas de doute, traiter tous les cas de paludisme à $P$. falciparum comme s'ils étaient résistants aux médicaments. 


\section{Prise en charge du paludisme grave ou compliqué}

Le paludisme grave ou compliqué ou encore l'incapacité à tolérer un traitement oral, exige un traitement urgent par voie parentérale et une prise en charge médicale intensive (particulièrement dans le cas des enfants), idéalement dans une unité de soins intensifs. Dans la mesure du possible, consultez un spécialiste en maladies tropicales ou infectieuses lorsqu'il s'agit de prendre en charge un patient atteint de paludisme à $P$. falciparum grave.

L'objectif principal du traitement consiste à éviter le décès du patient. La prévention des déficiences neurologiques est également un objectif important du traitement du paludisme cérébral. II existe plusieurs traitements :

- L'artésunate parentéral constitue le traitement de première intention du paludisme à $P$. falciparum grave recommandé par l'Organisation mondiale de la Santé.

- La quinine parentérale peut être utilisée pour traiter le paludisme grave ou compliqué lorsque l'artésunate parentéral n'est pas accessible. Elle est le médicament de choix si la seule indication pour le traitement parentéral est l'intolérance au traitement oral.

- L'artésunate ou la quinine parentéraux sont accessibles 24 heures sur 24 par l'entremise du Réseau canadien sur le paludisme (30).

- À la suite de l'administration parentérale de l'artésunate ou de la quinine, administrer un traitement oral par l'un des médicaments suivants :

- atovaquone/proguanil (à moins que ces médicaments aient été utilisés en tant que chimioprophylaxie antipaludéenne);

- doxycycline (à moins que ce médicament ait été utilisé en tant que chimioprophylaxie antipaludéenne; contre-indications : femmes enceintes ou qui allaitent et enfants de moins de 8 ans) (7);

- clindamycine (seulement si le patient est incapable de prendre la doxycycline ou l'association atovaquone/proquanil).

Le Tableau 2 présente les antipaludéens courants et leurs indications.

Tableau 2: Recommandations concernant les antipaludéens courants

\begin{tabular}{|c|c|c|}
\hline & Indication & Remarques additionnelles \\
\hline $\begin{array}{l}\text { Artésunate par voie } \\
\text { intraveineuse }\end{array}$ & $\begin{array}{l}\text { Traitement de première intention du } \\
\text { paludisme à } P \text {. falciparum }(3,7,31) \\
\text { grave ou en cas d'intolérance à la } \\
\text { quinine administrée par voie } \\
\text { intraveineuse. }\end{array}$ & $\begin{array}{l}\text { Administrer un traitement complet par } \\
\text { l'atovaquone/proguanil (Malarone }{ }^{\mathrm{MD}} \text { ), la } \\
\text { doxycycline (clindamycine chez les } \\
\text { femmes enceintes ou les enfants } \\
<8 \text { ans) ou une polythérapie à base } \\
\text { d'artémisinine à la suite de } \\
\text { l'administration parentérale de } \\
\text { l'artésunate. } \\
\text { Il faudrait effectuer un hémogramme } \\
\text { hebdomadairement pendant quatre } \\
\text { semaines aux patients qui reçoivent de } \\
\text { l'artésunate en intraveineuse. En outre, } \\
\text { ils devraient être encouragés à déclarer } \\
\text { des signes d'hémolyse : urine foncée, } \\
\text { jaunissement de la peau ou du blanc des } \\
\text { yeux, fièvre, douleur abdominale, pâleur, } \\
\text { fatigue, essoufflement ou douleurs } \\
\text { thoraciques. }\end{array}$ \\
\hline $\begin{array}{l}\text { Polythérapie orale par } \\
\text { l'artémisinine } \\
\text { (non encore accessible au }\end{array}$ & $\begin{array}{l}\text { Paludisme à } P \text {. falciparum non } \\
\text { compliqué ou lorsque l'espèce en cause } \\
\text { n'a pas été identifiée }\end{array}$ & \\
\hline
\end{tabular}




\begin{tabular}{|c|c|c|}
\hline & Indication & Remarques additionnelles \\
\hline \multicolumn{3}{|l|}{ Canada) } \\
\hline $\begin{array}{l}\text { Atovaquone/proguanil } \\
\text { Nom commercial : } \\
\text { Malarone }\end{array}$ & $\begin{array}{l}\text { Traitement de première intention du } \\
\text { paludisme aigu non compliqué à } \\
P . \text { falciparum ou à } P \text {. vivax } \\
\text { Paludisme non compliqué chez les } \\
\text { adultes et les enfants } \geq 11 \mathrm{~kg} \text {. }\end{array}$ & \\
\hline $\begin{array}{l}\text { Chloroquine (ou } \\
\text { hydroxychloroquine) } \\
\text { Nom commercial : Novo- } \\
\text { Chloroquine (ou Plaquenil } \\
\text { Apo-Hydroxyquine, Gen- } \\
\text { Hydroxychloroquine) }\end{array}$ & $\begin{array}{l}\text { Infections à } P . \text { falciparum, à } P . \text { vivax, à } \\
P . \text { ovale, à } P \text {. malariae ou à } P . \text { knowlesi } \\
\text { sensibles à la chloroquine. }\end{array}$ & $\begin{array}{l}\text { Convient aux sujets de tous les âges et } \\
\text { aux femmes enceintes. }\end{array}$ \\
\hline $\begin{array}{l}\text { Clindamycine } \\
\text { Nom commercial : } \\
\text { (Dalacin C } \\
\text { Clindamycin, Novo- } \\
\text { Clindamycin }\end{array}$ & $\begin{array}{l}\text { La clindamycine est administrée en } \\
\text { association avec la quinine pour traiter le } \\
\text { paludisme à } P \text {. falciparum résistant à la } \\
\text { chloroquine ou à la méfloquine chez les } \\
\text { femmes enceintes, les enfants (< } 8 \text { ans) } \\
\text { et les adultes intolérants à la tétracycline } \\
\text { lorsqu'il est impossible d'avoir accès à } \\
\text { des dérivés de l'artémisinine. }\end{array}$ & $\begin{array}{l}\text { Moins efficace que la doxycycline ou } \\
\text { l'association atovaquone/proguanil. }\end{array}$ \\
\hline $\begin{array}{l}\text { Doxycycline } \\
\text { Nom commercial : Vibra- } \\
\text { Tabs }{ }^{\mathrm{MD}} \text {, Apo-Doxy, Doxycin, } \\
\text { Novo-Doxylin, Nu- } \\
\text { Doxycycline, ratio- } \\
\text { Doxycycline }\end{array}$ & $\begin{array}{l}\text { Pour prévenir et traiter le paludisme à } \\
P . \text { falciparum résistant à la chloroquine. }\end{array}$ & $\begin{array}{l}\text { Contre-indications : Femmes enceintes } \\
\text { ou qui allaitent et enfants }<8 \text { ans. }\end{array}$ \\
\hline Quinine et quinidine & $\begin{array}{l}\text { La quinine parentérale est utilisée pour } \\
\text { traiter le paludisme grave ou compliqué } \\
\text { lorsque l'artésunate parentérale n'est pas } \\
\text { accessible et comme médicament de } \\
\text { première intention chez les sujets qui ne } \\
\text { peuvent pas recevoir le traitement oral } \\
\text { ou qui ne satisfont pas aux critères du } \\
\text { paludisme grave. } \\
\text { Le traitement oral par la quinine en } \\
\text { association avec la doxycycline ou la } \\
\text { clindamycine est indiqué dans le cas du } \\
\text { paludisme à } P \text {. falciparum non compliqué } \\
\text { et comme traitement dégressif à la suite } \\
\text { d'un traitement parentéral du paludisme } \\
\text { compliqué. }\end{array}$ & $\begin{array}{l}\text { Le Réseau canadien sur le paludisme } \\
\text { recommande de limiter l'utilisation de } \\
\text { l'artésunate aux patients atteints de } \\
\text { paludisme grave (tel qu'il est défini par } \\
\text { l'Organisation mondiale de la Santé) et } \\
\text { d'utiliser la quinine parentérale chez les } \\
\text { sujets intolérants à la thérapie orale ou } \\
\text { ceux chez qui ce traitement provoque } \\
\text { des vomissements. } \\
\text { La quinidine parentérale ne doit être } \\
\text { utilisée que si les deux médicaments de } \\
\text { première intention ne sont pas } \\
\text { disponibles. Une surveillance de la } \\
\text { fonction cardiaque est requise. }\end{array}$ \\
\hline $\begin{array}{l}\text { Primaquine } \\
\text { Nom commercial : } \\
\text { Primaquine (phosphate de } \\
\text { primaquine) }\end{array}$ & $\begin{array}{l}\text { Utilisée pour prévenir les rechutes } \\
\text { d'infections à } P \text {. vivax ou à } P \text {. ovale. } \\
\text { Utilisée comme «traitement radical " } \\
\text { pour réduire le risque de rechute après le } \\
\text { traitement d'une infection symptomatique } \\
\text { à } P \text {. vivax ou à } P \text {. ovale. }\end{array}$ & $\begin{array}{l}\text { Contre-indiquée chez les sujets } \\
\text { présentant un déficit grave en G-6-PD, } \\
\text { les femmes enceintes et les femmes qui } \\
\text { allaitent si le nourrisson présente un } \\
\text { déficit en G-6-PD. }\end{array}$ \\
\hline
\end{tabular}

Si la quinine ou l'artésunate parentéraux sont indiqués mais que l'on ne peut y avoir accès dans un délai d'une heure, un traitement oral par la quinine doit être amorcé (après l'administration d'une dose de Gravol) ou encore de la quinine peut être administrée par une sonde nasogastrique en attendant le traitement parentéral. Les patients doivent recevoir le traitement parentéral pendant au moins 24 heures avant de passer au traitement oral. 
L'état du malade devrait être observé fréquemment pour surveiller les signes vitaux et évaluer la fréquence et le profil respiratoires, le score de coma et le débit urinaire. Utiliser des tests rapides (bâtonnets) pour mesurer la glycémie au moins à toutes les quatre heures. Traiter rapidement les crises d'épilepsie par des benzodiazépines (7). Réaliser chaque jour une évaluation clinique de tous les patients jusqu'à la disparition de la fièvre et chaque fois qu'il y a récurrence des symptômes. Dans les cas de paludisme à $P$. falciparum, effectuer chaque jour des frottis jusqu'à ce que les résultats soient négatifs.

Lorsque le paludisme s'accompagne d'une forte parasitémie ( $\geq 10 \%)$, une transfusion d'échange employée pour éliminer les érythrocytes infectés ou les médiateurs toxiques de la circulation et pour réduire la charge parasitaire peut se révéler bénéfique $(8,32)$.

\section{Prise en charge du paludisme à $P$. falciparum non compliqué}

On entend par paludisme non compliqué un paludisme symptomatique qui ne s'accompagne d'aucun signe de maladie grave ou de dysfonctionnement d'organes vitaux. Le traitement du paludisme non compliqué vise à guérir l'infection et à prévenir la progression vers la maladie grave. Lorsqu'on choisit des régimes thérapeutiques, il convient de tenir compte de la tolérabilité au médicament, du profil d'effets indésirables et de la rapidité de la réponse thérapeutique.

Les traitements de choix du paludisme à $P$. falciparum non compliqué consistent en l'administration de :

- chloroquine par voie orale (SEULEMENT chez les sujets ayant voyagé dans une région où les souches sont sensibles uniquement à la chloroquine);

- atovaquone/proguanil par voie orale (7);

- quinine par voie orale administrée en association avec la doxycycline par voie orale soit en même temps, soit l'une après l'autre (en commençant par la quinine); si la doxycycline est contre-indiquée, administrer la quinine et la clindamycine par voie orale soit en même temps, soit l'une après l'autre (33-34);

- polythérapie par un dérivé de l'artémisinine (non encore accessible au Canada) (7).

\section{Prise en charge du paludisme dû à une espèce autre que $P$. falciparum}

Réaliser chaque jour une évaluation clinique jusqu'à la disparition de la fièvre et chaque fois qu'il y a récurrence des symptômes. Dans le cas des infections à $P$. vivax, une récurrence de la parasitémie asexuée moins de 30 jours après le traitement indique qu'il s'agit d'une infection à $P$. vivax chloroquino-résistante; une récurrence après 30 jours évoque une résistance de $P$. vivax à la primaquine.

La chloroquine demeure le traitement de choix pour le paludisme causé par une espèce autre que $P$. falciparum dans les régions où cette souche n'est pas résistante à la chloroquine. On ignore en quoi consiste la chimioprophylaxie ou le traitement optimal du paludisme à $P$. vivax contracté dans une région où la souche est résistante à la chloroquine, bien qu'un traitement de sept jours par la quinine soit souvent requis pour guérir l'infection à $P$. vivax.

\section{Prise en charge du paludisme dû à une espèce non identifiée}

Si la présence de fièvre, les antécédents de voyages et les données initiales de laboratoire (faible numération leucocytaire ou plaquettaire) évoquent un diagnostic de paludisme et si le frottis sanguin est retardé plus de deux heures, il convient d'amorcer un traitement antipaludéen efficace en fonction de la région où l'infection a été contractée ou du type de paludisme contracté.

\section{Prise en charge des rechutes}

$P$. vivax et $P$. ovale ont un stade hépatique persistant (hypnozoïtes) qui est à l'origine des rechutes pouvant survenir pendant des mois et même des années après l'exposition, même en l'absence d'une infection symptomatique primaire au paludisme. Aucun des régimes chimioprophylactiques actuellement recommandés ne permet de prévenir les rechutes causées par $P$. vivax ou $P$. ovale. 
Afin de réduire le risque de rechute après le traitement d'une infection symptomatique à $P$. vivax ou à $P$. ovale, la primaquine est indiquée comme « traitement radical ». Une fois la maladie fébrile aiguë disparue, amorcer le traitement radical par la primaquine de manière à ce qu'il chevauche le traitement par un schizonticide sanguin (c.-à-d. chloroquine ou quinine) (7).

La primaquine est contre-indiquée chez les sujets présentant un déficit grave en G-6-PD et chez les femmes enceintes. On peut prévenir les rechutes chez les femmes enceintes par l'administration de doses hebdomadaires de chloroquine jusqu'à l'accouchement. Par la suite, on peut prescrire sans danger de la primaquine aux mères dont le taux de G-6-PD est normal, à moins qu'elles allaitent. Les mères qui allaitent ne peuvent recevoir la primaquine que si le nourrisson a été soumis à des examens et que les résultats indiquent qu'il ne présente aucun déficit en G-6-PD.

P. knowlesi, qui représente une menace en Asie du Sud-Est, peut être confondue au microscope avec P. malariae, exception faite de son taux de parasitémie plus élevé (> $1 \%)$. Les complications et les symptômes généraux peuvent ressembler à ceux du paludisme à $P$. falciparum. Le traitement par la chloroquine serait efficace, semble-t-il, mais il faut surveiller très étroitement et traiter soigneusement les complications et les symptômes généraux similaires à ceux des infections hyperparasitémiques à $P$. falciparum $(25,35)$, et administrer possiblement un traitement parentéral par l'artésunate.

\section{Auto-traitement en cas de paludisme présumé}

L'auto-traitement est une mesure salvatrice à prendre dans un délai de 24 heures pendant qu'on tente d'obtenir l'aide d'un médecin. Les personnes qui se rendent dans une région présentant un risque élevé (p. ex. en Afrique subsaharienne où surviennent $90 \%$ de tous les cas de morbidité et de mortalité associés au paludisme) ne devraient pas compter uniquement sur l'auto-traitement $(9,21,23,25)$. Les signes et symptômes du paludisme ne sont pas particuliers, et il est impossible de poser un diagnostic définitif de paludisme en l'absence d'une analyse de laboratoire $(10,37,38)$.

Les voyageurs exposés à un risque de paludisme qui ne peuvent pas consulter un médecin dans les 24 heures afin de recevoir un traitement antipaludéen adéquat devraient apporter des médicaments efficaces pour l'autotraitement du paludisme présumé.

- Dans les régions où le paludisme est sensible à la chloroquine, il convient d'amorcer l'auto-traitement à la chloroquine, puis continuer ou amorcer une prophylaxie à la chloroquine (9-11).

- Dans les régions où $P$. falciparum est résistante à la chloroquine ou à la chloroquine et à la méfloquine, l'auto-traitement devrait consister en l'administration d'un médicament différent de celui utilisé en tant qu'agent chimioprophylactique. Idéalement, ce médicament devrait avoir été acheté dans un pays où les normes de contrôle de la qualité sont rigoureuses afin de réduire au minimum le risque d'utiliser un produit contrefait. On choisira l'une des options suivantes :

$\circ$ atovaquone/proguanil

$\circ$ quinine et doxycycline orales $(10-11,13)$

○ artéméther/luméfantrine

\section{Antipaludéens contre-indiqués}

Un certain nombre d'antipaludéens sont contre-indiqués pour le traitement du paludisme (auto-traitement ou autre) :

- méfloquine;

- $\quad$ sulfadoxine/pyriméthamine (Fansidar);

- méfloquine/Fansidar;

- halofantrine;

- chloroquine/Fansidar. 


\section{Résumé}

Le Tableau 3 résume les principaux changements apportés aux lignes directrices de 2014.

Tableau 3 : Résumé des principaux ajouts et changements aux lignes directrices de 2014 concernant le diagnostic et le traitement du paludisme (4)

\section{Ajouts}

1. On a mis à jour les directives sur la prise en charge du paludisme grave pour inclure de nouveaux renseignements sur le recours à une transfusion d'échange (voir le chapitre 7).

2. Une nouvelle fiche sur le paludisme peut être donnée aux voyageurs pour leur fournir des renseignements sur leur chimioprophylaxie antipaludéenne tout en leur rappelant de consulter un médecin en cas de fièvre après un voyage.

\section{Changements}

1. Le chapitre 8, « Médicaments pour la prévention et le traitement du paludisme », comprend des renseignements actualisés sur la posologie pour les enfants de l'atovaquone et du proguanil ainsi que des mises à jour générales au tableau 8.11: Médicaments (nom générique et commercial) pour la prévention et le traitement du paludisme. On a aussi révisé les sous-sections portant sur l'artémisinine, la chloroquine ainsi que la quinine et la quinidine.

2. On a modifié le taux de parasitémie utilisé pour définir les cas de paludisme grave hyperparasitémique; il est maintenant fixé entre $\geq 2 \%$ et $5 \%$ chez les voyageurs non immuns.

\section{Conclusion}

La prise en charge du paludisme repose sur l'identification rapide de la maladie ainsi que sur l'identification de l'espèce de plasmodies et le dosage de la parasitémie. Toute personne atteinte de paludisme grave ou compliqué doit être hospitalisée. Le traitement du paludisme non compliqué vise à guérir l'infection et à prévenir la progression vers la maladie grave. Le traitement varie selon l'espèce de plasmodies en cause, la gravité du paludisme et la région où la maladie a été contractée.

\section{Remerciements}

Le CCMTMV tient à remercier Joanna Odrowaz et Elspeth Payne pour leur contribution à l'élaboration des sommaires et Manisha Kulkarni pour sa contribution à la déclaration.

Membres du CCMTMV : Boggild A., Brophy J., Bui Y. G., Crockett M., Ghesquiere W., Greenaway C., Henteleff A., Libman M., Teitelbaum P. et McCarthy A. (présidente).

Représentants chargés de la liaison : Hui C. (Société canadienne de pédiatrie) et Gershman M. (Centers for Disease Control and Prevention [É.-U.]).

Membres d'office : Marion D. (Centre des services de santé des Forces canadiennes, ministère de la Défense nationale), McDonald P. (Division des médicaments anti-infectieux, Santé Canada), Schofield S. (Direction de la protection de la santé de la Force, ministère de la Défense nationale) et Tepper M. (Direction de la protection de la santé de la Force, ministère de la Défense nationale).

Membre émérite : Jeanes C. W. L. 


\section{Conflit d'intérêts}

II n'y a aucun conflit d'intérêts à déclarer.

\section{Financement}

Ce travail a été appuyé par l'Agence de la santé publique du Canada. 


\section{Références}

(1) McCarthy AE, Plourde P, Kuhn S et Bodie M. Parenteral quinine for severe malaria: five year surveillance data from the Canadian Malaria Network. 10th Conference of the International Society of Travel Medicine 2007; Abstract No. FC02.01.

(2) Murphy GS et Oldfield EC, 3rd. Falciparum malaria. Infect Dis Clin North Am. Déc. 1996;10(4):747-775.

(3) Sinclair D, Donegan S et Lalloo DG. Artesunate versus quinine for treating severe malaria. Cochrane Database Syst Rev. 16 mars 2011;(3):CD005967. doi(3):CD005967.

(4) Comité consultatif de la médecine tropicale et de la médecine des voyages. Recommandations canadiennes pour la prévention et le traitement du Paludisme (Malaria) (sous presse).

http://publications.gc.ca/site/fra/463471/publication.html

(5) Comité consultatif de la médecine tropicale et de la médecine des voyages. Summary of recommendations for the prevention of malaria by the Committee to Advise on Tropical Medicine and Travel (CATMAT). Relevé des maladies transmissibles au Canada, vol. 40, $\mathrm{n}^{\circ}$ 7, 2014.

(6) Leslie T, Mayan I, Mohammed N, Erasmus P, Kolaczinski J, Whitty CJ et al. A randomised trial of an eightweek, once weekly primaquine regimen to prevent relapse of Plasmodium vivax in Northwest Frontier Province, Pakistan. PLoS One. Août 2008;3(8):e2861.

(7) Organisation mondiale de la Santé. Directives pour le traitement du paludisme, deuxième édition. 2010.

(8) Riddle MS, Jackson JL, Sanders JW et Blazes DL. Exchange transfusion as an adjunct therapy in severe Plasmodium falciparum malaria: a meta-analysis. Clin Infect Dis. Mai 2002;34(9):1192-1198.

(9) Schlagenhauf P et Petersen E. Standby emergency treatment of malaria in travelers: experience to date and new developments. Expert Rev Anti Infect Ther. Mai 2012;10(5):537-546.

(10) Nothdurft HD, Jelinek T, Pechel SM, Hess F, Maiwald H, Marschang A et al. Stand-by treatment of suspected malaria in travellers. Trop Med Parasitol. Sept. 1995;46(3):161-163.

(11) Schlagenhauf P et Steffen R. Stand-by treatment of malaria in travellers: a review. J Trop Med Hyg. Juin 1994;97(3):151-160.

(12) Newton P, Proux S, Green M, Smithuis F, Rozendaal J, Prakongpan S et al. Fake artesunate in southeast Asia. Lancet. Juin 2001357(9272):1948-1950.

(13) Cockburn R, Newton PN, Agyarko EK, Akunyili D et White NJ. The global threat of counterfeit drugs: why industry and governments must communicate the dangers. PLoS Med. Avril 2005;2(4):e100.

(14) Prasad K et Garner P. Steroids for treating cerebral malaria. Cochrane Database Syst Rev 2000;(2):CD000972.

(15) Weinke T, Trautmann M, Held T, Weber G, Eichenlaub D, Fleischer K et al. Neuropsychiatric side effects after the use of mefloquine. Am J Trop Med Hyg. Juil. 1991;45(1):86-91.

(16) Roll Back Malaria Department, WHO et UNICEF. World Malaria Report. 2005.

(17) Luxemburger C, Price RN, Nosten F, Ter Kuile FO, Chongsuphajaisiddhi T et White NJ. Mefloquine in infants and young children. Ann Trop Paediatr. Déc. 1996;16(4):281-286.

(18) Chen LH, Wilson ME et Schlagenhauf P. Controversies and misconceptions in malaria chemoprophylaxis for travelers. JAMA. Mai 2007;297(20):2251-2263. 
(19) Mali S, Kachur SP et Arguin PM. Division of Parasitic Diseases and Malaria, Center for Global Health, Centers for Disease Control and Prevention (CDC). Malaria surveillance - United States, 2010. MMWR Surveill Summ Mars 2012;61(2):1-17.

(20) Svenson JE, Gyorkos TW et MacLean JD. Diagnosis of malaria in the febrile traveler. Am J Trop Med Hyg 1995;53(5):518-521.

(21) Swales CA, Chiodini PL et Bannister BA, Health Protection Agency Advisory Committee on Malaria Prevention in UK Travellers. New guidelines on malaria prevention: a summary. J Infect. Févr. 2007;54(2):107-110.

(22) Reyburn H, Mbatia R, Drakeley C, Carneiro I, Mwakasungula E, Mwerinde O et al. Overdiagnosis of malaria in patients with severe febrile illness in Tanzania: a prospective study. BMJ. Nov. 2004;329(7476):1212.

(23) Quach C, Kain K, MacPherson D, Mendelson J et MacLean J. Malaria deaths in Canadian travellers. Can Commun Dis Rep. Mars 1999;25(6):50-53.

(24) Cox-Singh J, Davis TM, Lee KS, Shamsul SS, Matusop A, Ratnam S et al. Plasmodium knowlesi malaria in humans is widely distributed and potentially life threatening. Clin Infect Dis. Janv. 2008;46(2):165-171.

(25) Centers for Disease Control and Prevention (CDC). CDC Health Information for International Travel 2012. New York: Oxford University Press; 2012.

(26) Chen LH et Wilson ME, Schlagenhauf P. Prevention of malaria in long-term travelers. JAMA. Nov. 2006;296(18):2234-2244.

(27) Farcas GA, Soeller R, Zhong K, Zahirieh A et Kain KC. Real-time polymerase chain reaction assay for the rapid detection and characterization of chloroquine-resistant Plasmodium falciparum malaria in returned travelers. Clin Infect Dis. Mars 2006;42(5):622-627.

(28) Hawkes M et Kain KC. Advances in malaria diagnosis. Expert Rev Anti Infect Ther. Juin 2007;5(3):485-495.

(29) Farcas GA, Zhong KJ, Mazzulli T et Kain KC. Evaluation of the RealArt Malaria LC real-time PCR assay for malaria diagnosis. J Clin Microbiol. Fév. 2004;42(2):636-638.

(30) Agence de la santé publique du Canada. Artésunate et quinine dans le traitement du paludisme : accès médical simplifié au Canada par l'entremise du Réseau canadien sur le paludisme. Accès : http://www.phacaspc.gc.ca/tmp-pmv/quinine/index-fra.php [consulté le 6 mars 2014].

(31) Dondorp A, Nosten F, Stepniewska K, Day N et White N. South East Asian Quinine Artesunate Malaria Trial (SEAQUAMAT) group. Artesunate versus quinine for treatment of severe falciparum malaria: a randomised trial. Lancet. 27 août-2 sept. 2005;366(9487):717-725.

(32) Nieuwenhuis JA, Meertens JH, Zijlstra JG, Ligtenberg JJ, Tulleken JE et van der Werf TS. Automated erythrocytapheresis in severe falciparum malaria: a critical appraisal. Acta Trop. Juil. 2006;98(3):201-206.

(33) Organisation mondiale de la Santé. Voyages internationaux et santé. Genève (Suisse) : Organisation mondiale de la Santé; 2012.

(34) Griffith KS, Lewis LS, Mali S et Parise ME. Treatment of malaria in the United States: a systematic review. JAMA. Mai 2007;297(20):2264-2277.

(35) White NJ. Plasmodium knowlesi: the fifth human malaria parasite. Clin Infect Dis. Janv. 2008;46(2):172-173.

(36) Singh B, Kim Sung L, Matusop A, Radhakrishnan A, Shamsul SS, Cox-Singh J et al. A large focus of naturally acquired Plasmodium knowlesi infections in human beings. Lancet. Mars 2004;363(9414):1017-1024. 
(37) Schlagenhauf P, Steffen R, Tschopp A, Van Damme P, Mittelholzer ML, Leuenberger H et al. Behavioural aspects of travellers in their use of malaria presumptive treatment. Bull World Health Organ 1995; 73(2):215-221.

(38) Organisation mondiale de la Santé. Comité OMS d'experts du paludisme : vingtième rapport. Genève (Suisse) : Organisation mondiale de la Santé; 2000. Rapport $n^{\circ} 892$. 